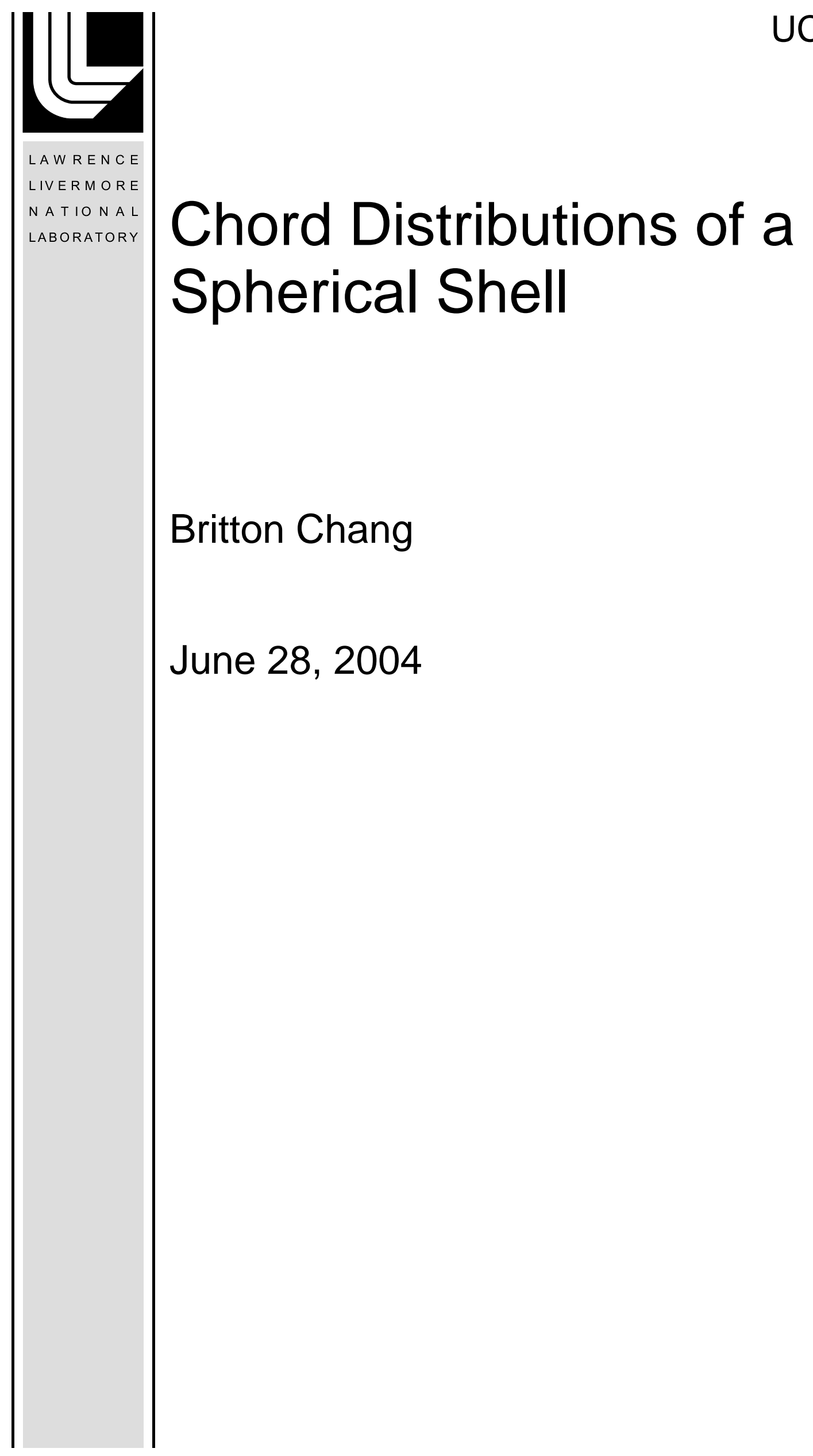


This document was prepared as an account of work sponsored by an agency of the United States Government. Neither the United States Government nor the University of California nor any of their employees, makes any warranty, express or implied, or assumes any legal liability or responsibility for the accuracy, completeness, or usefulness of any information, apparatus, product, or process disclosed, or represents that its use would not infringe privately owned rights. Reference herein to any specific commercial product, process, or service by trade name, trademark, manufacturer, or otherwise, does not necessarily constitute or imply its endorsement, recommendation, or favoring by the United States Government or the University of California. The views and opinions of authors expressed herein do not necessarily state or reflect those of the United States Government or the University of California, and shall not be used for advertising or product endorsement purposes.

This work was performed under the auspices of the U.S. Department of Energy by University of California, Lawrence Livermore National Laboratory under Contract W-7405-Eng-48. 


\title{
CHORD DISTRIBUTIONS OF A SPHERICAL SHELL
}

\author{
BRITTON CHANG *
}

1. Introduction. Many years ago, Dirac [1], [2] derived an approximation for $\alpha$, the eigenvalue of the Neutron Transport Equation which is physically the criticality of a chunk of nuclear material, in terms of the distribution of the chord lengths in the chunk. The criticality of an assemblage of nuclear material is linked to the chord lengths of the assembly, because the net number of secondary neutrons which is produced in a collision by a primary neutron with an atom of the fissionable medium is proportional to the number of mean free paths that the primary neutron can travel in the assembly. Dirac derived the chord length distributions for three convex solids; an oblate ellipsoid, a hemi-sphere, and a hemi-ellipsoid.

In this paper, we derive the distributions for two kinds of chords for a spherical shell. The first kind are truncated chords which are drawn in the left figure of Fiq. 1. The second kind are complete chords which are drawn in the right figure of Fig. 1. Truncated chords are chords which originate at a point $r$ and terminate at the nearest boundary of the shell. We can think of a truncated chord as the path in which a particle is born at point $r$ and travels in the direction $\mu$ to the nearest boundary of the shell. For the case of the truncated chords, we can think of the region bounded by the inner surface of the shell as being filled with a perfect absorber. If the particle leaves the shell through the inner surface, it is absorbed by the perfect absorber. On the other hand, complete chords are straight lines that spans the sphere as if inner void region of the shell was not there. If a complete chord leaves the shell through the inner surface, it re-enters the shell through the other side of the void. The length of a complete chord is the length of the portion of the straight line that is within the shell.

2. The Distribution of Truncated Chord Lengths of a Spherical Shell. If the inner and outer radii of a spherical shell are $a$ and $b$ respectively, then the volume of the spherical shell is $V=\frac{4 \pi}{3}\left(b^{3}-a^{3}\right)$. If we assume that the interior points of the spherical shell are equally probable, then the interior points are distributed according to the distribution law

$$
\rho_{r}=\frac{4 \pi r^{2}}{V}=\frac{3 r^{2}}{b^{3}-a^{3}}, \quad a \leq r \leq b .
$$

It is easy to verify that $\int_{a}^{b} d r \rho_{r}=1$. Furthermore let $\mu$ be the cosine of the angle that is between the radius vector and the direction of the path of a neutron. If we assume that at each interior point $r$, the paths through this point is isotropically distributed, then the 'angular' distribution law of the paths is

$$
\rho_{\mu}=\frac{1}{2}, \quad-1 \leq \mu \leq 1
$$

Since the joint probability density is the product of $\rho_{r}$ and $\rho_{\mu}$, then the conditional probability density of the angle, given $r$, is $\rho(\mu \mid r)=\rho_{\mu}=1 / 2$.

${ }^{*}$ Center for Applied Scientific Computing, Lawrence Livermore National Laboratory, P.O. Box 808 L-561, Livermore, CA 94551. email: bchang@llnl.gov. This work was performed under the auspices of the U.S. Department of Energy by Lawrence Livermore National Laboratory under contract no. W-7405-Eng-48. 

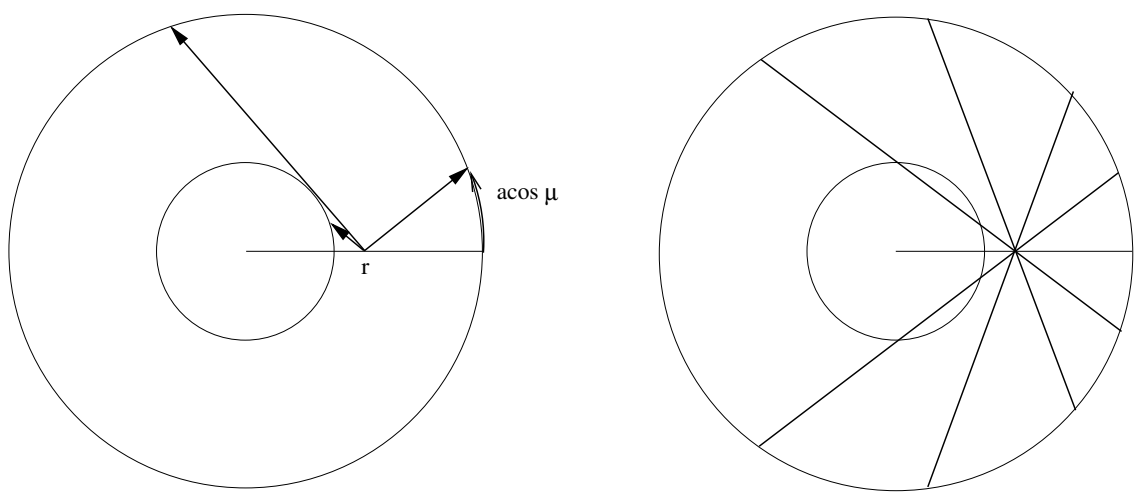

FIG. 2.1. The left figure illustrates truncated chords in a spherical shell, and the right figure illustrates complete chords in a spherical shell.

The derivation of the distribution of path lengths $\rho_{l}$ from the joint probability density $\rho_{r} \rho(\mu \mid r)$ is an excercise in the change of integration variables from $r$ and $\mu$ to $r$ and $l$, where $l$ is the length of the path from the point $r$ in the direction $\mu$ to the nearest boundary of the shell. The left figure in Fig. 1 shows that there are two types of truncated chords which emanate from the point $r$ in a spherical shell. The first type of path is from the point $r$ to the inner wall of the shell, while the second type of path is from the point $r$ the outer wall of the shell. It is clear from the left figure of Fig. 1 that the path length $l$ is discontinous with respect to the angle $\mu$. At the angle of discontinuity $\mu=-\sqrt{1-a^{2} / r^{2}}$, the path grazes the inner boundary. This discontinuity adds a small degree of difficulty to the change of variables, but this small difficulty is easy to overcome.

In order to keep the presentation simple, let us define the integral which is the focus of our attention as

$$
\begin{aligned}
Q & \equiv \int_{a}^{b} d r \rho_{r} \int_{-1}^{1} d \mu \rho(\mu \mid r) \\
& =\int_{a}^{b} d r \rho_{r} \int_{-1}^{-\frac{\sqrt{r^{2}-a^{2}}}{r}} d \mu \rho(\mu \mid r)+\int_{a}^{b} d r \rho_{r} \int_{-\frac{\sqrt{r^{2}-a^{2}}}{r}}^{1} d \mu \rho(\mu \mid r),
\end{aligned}
$$

where the $\mu$ integral is divided into two pieces in order to account for the two types of paths mentioned above. If we define the $\mu$ integrals of the above equation as

$$
\begin{aligned}
& P_{a}(r) \equiv \int_{-1}^{-\frac{\sqrt{r^{2}-a^{2}}}{r}} d \mu \rho(\mu \mid r), \\
& P_{b}(r) \equiv \int_{-\frac{\sqrt{r^{2}-a^{2}}}{r}}^{1} d \mu \rho(\mu \mid r),
\end{aligned}
$$

then $Q$ of (2.3) can be written as $Q=Q_{a}+Q_{b}$, where

$$
\begin{aligned}
Q_{a} & \equiv \int_{a}^{b} d r \rho_{r} P_{a}(r), \\
Q_{b} & \equiv \int_{a}^{b} d r \rho_{r} P_{b}(r) .
\end{aligned}
$$




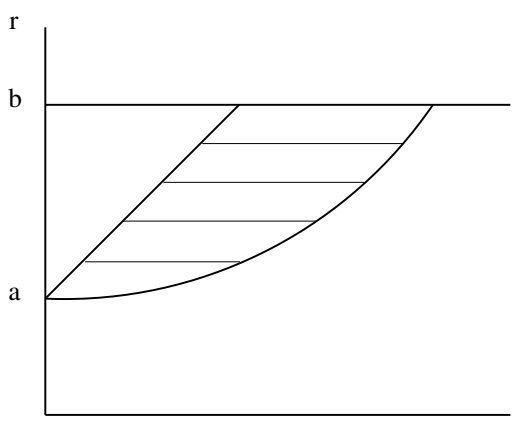

1

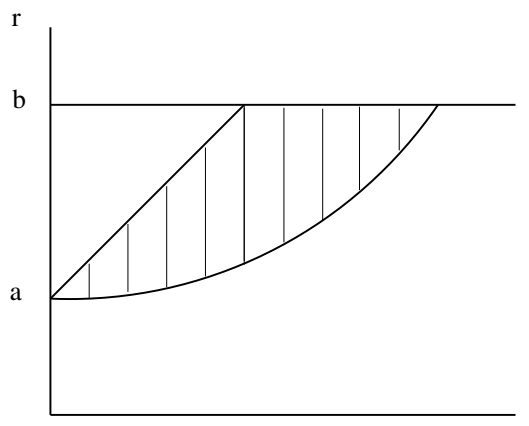

1

FIG. 2.2. The domain of integration of $Q_{a}$ can be decomposed in two ways. The figure on the left describes the double integral as holding $r$ fixed while integrating over $l$. The figure on the right describes the double integral as holding $l$ fixed while integrating over $r$.

The paths of (2.4) are those that intersect inner wall of the shell. The lengths of these paths can be determined simply by geometry, and are

$$
l_{a}=-\sqrt{a^{2}-\left(1-\mu^{2}\right) r^{2}}-\mu r .
$$

The integration limit $\mu=-1$ corresponds to the path length $l_{a}=r-a$, and the integration limit $\mu=-\sqrt{1-a^{2} / r^{2}}$ corresponds to the path length $l_{a}=\sqrt{r^{2}-a^{2}}$. Since we wish to express the integral in (2.4) in terms of $l_{a}$, we solve (2.8) for $\mu$, i.e.

$$
\mu=\frac{a^{2}-r^{2}-l_{a}^{2}}{2 r l_{a}},
$$

from which we get

$$
\frac{d \mu}{d l_{a}}=\frac{r^{2}-a^{2}-l_{a}^{2}}{2 r l_{a}^{2}} .
$$

The substitution of (2.10) into (2.4) gives

$$
P_{a}(r)=\int_{r-a}^{\sqrt{r^{2}-a^{2}}} \rho(\mu \mid r) \frac{d \mu}{d l_{a}} d l_{a}=\int_{r-a}^{\sqrt{r^{2}-a^{2}}} \frac{r^{2}-a^{2}-l^{2}}{4 r l^{2}} d l
$$

where the subscript $a$ on $l_{a}$ is dropped in the last integral since $l_{a}$ is a dummy integration variable.

Now the substitution of (2.11) into (2.6) gives

$$
Q_{a}=\int_{a}^{b} d r \rho_{r} \int_{r-a}^{\sqrt{r^{2}-a^{2}}} \frac{r^{2}-a^{2}-l^{2}}{4 r l^{2}} d l .
$$

We would like to change the order of integration in (2.12). Figure 2 shows the domain of integration and the limits of the double integral of (2.12). When the order of integration is reversed, Fig. 2 shows that the integral of (2.12) can be writtten as

$$
\begin{aligned}
Q_{a} & =\int_{0}^{b-a} d l \int_{\sqrt{a^{2}+l^{2}}}^{a+l} d r \rho_{r} \frac{r^{2}-a^{2}-l^{2}}{4 r l^{2}} \\
& +\int_{b-a}^{\sqrt{b^{2}-a^{2}}} d l \int_{\sqrt{a^{2}+l^{2}}}^{b} d r \rho_{r} \frac{r^{2}-a^{2}-l^{2}}{4 r l^{2}} .
\end{aligned}
$$


Since the $r$ integrals of (2.13) are analytical, then $Q_{a}$ can be written as

$$
Q_{a}=\int_{0}^{\sqrt{b^{2}-a^{2}}} d l \rho_{a}(l)
$$

where

$$
\rho_{a}(l)= \begin{cases}\frac{3 a^{2}}{4\left(b^{3}-a^{3}\right)}, & 0 \leq l \leq(b-a) \\ \frac{3\left(b^{2}-a^{2}-l^{2}\right)^{2}}{16\left(b^{3}-a^{3}\right) l^{2}}, & (b-a) \leq l \leq \sqrt{b^{2}-a^{2}},\end{cases}
$$

Turning to the integral $P_{b}$ of (2.5). The paths in (2.5) are those that intersect the outer wall of the shell. The lengths of these paths can also be determined simply by geometry, and are

$$
l_{b}=\sqrt{b^{2}-\left(1-\mu^{2}\right) r^{2}}-\mu r .
$$

We can solve (2.16) for $\mu$ to give

$$
\mu=\frac{b^{2}-r^{2}+l_{b}^{2}}{2 r l_{b}},
$$

from which we get

$$
\frac{d \mu}{d l_{b}}=-\frac{b^{2}-r^{2}-l_{b}^{2}}{2 r l_{b}^{2}} .
$$

The substitution of (2.18) into (2.5) gives

$$
P_{b}(r)=\int_{b-r}^{\sqrt{b^{2}-a^{2}}+\sqrt{r^{2}-a^{2}}} \rho(\mu \mid r) \frac{d \mu}{d l_{b}} d l_{b}=\int_{b-r}^{\sqrt{b^{2}-a^{2}}+\sqrt{r^{2}-a^{2}}} \frac{b^{2}-r^{2}+l^{2}}{4 r l^{2}} d l
$$

where the subscript $b$ on $l_{b}$ is dropped in the last integral since $l_{b}$ is a dummy integration variable.

The substitution of (2.19) into (2.7) gives

$$
Q_{b}=\int_{a}^{b} d r \rho_{r} \int_{b-r}^{\sqrt{b^{2}-a^{2}}+\sqrt{r^{2}-a^{2}}} \frac{b^{2}-r^{2}-l^{2}}{4 r l^{2}} d l .
$$

Figure 3 shows the domain of integration and the limits of the double integral of (2.20). When the order of integration is reversed, we can write the integral of (2.20) as

$$
\begin{aligned}
Q_{b} & =\int_{0}^{b-a} d l \int_{b-l}^{b} d r \rho_{r} \frac{b^{2}-r^{2}-l^{2}}{4 r l^{2}} \\
& +\int_{b-a}^{\sqrt{b^{2}-a^{2}}} d l \int_{a}^{b} d r \rho_{r} \frac{b^{2}-r^{2}-l^{2}}{4 r l^{2}} \\
& +\int_{\sqrt{b^{2}-a^{2}}}^{2 \sqrt{b^{2}-a^{2}}} d l \int_{\sqrt{\left(l-\sqrt{b^{2}-a^{2}}\right)^{2}+a^{2}}}^{b} d r \rho_{r} \frac{b^{2}-r^{2}-l^{2}}{4 r l^{2}}
\end{aligned}
$$



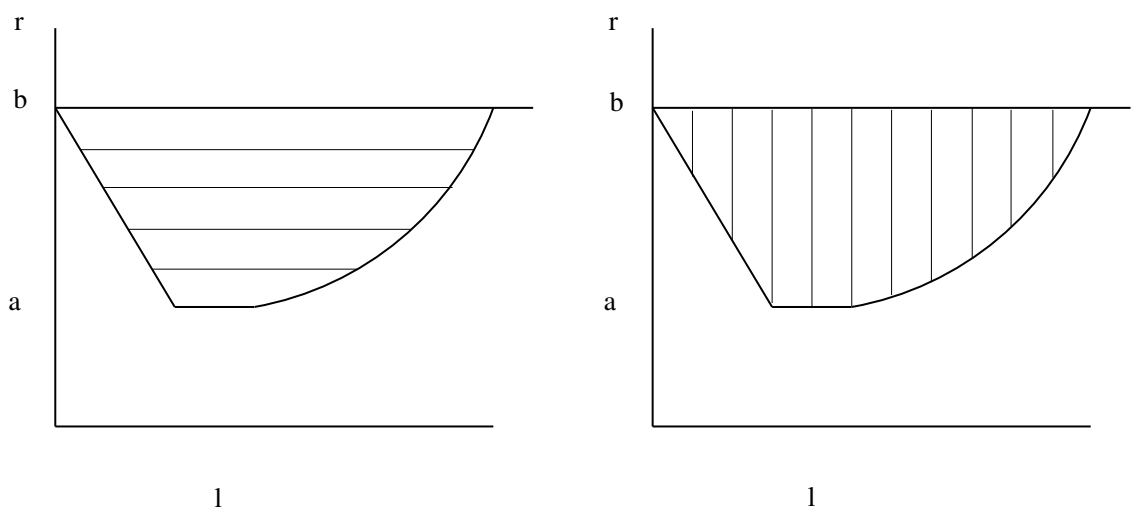

FIG. 2.3. The domain of integration of $Q_{b}$ can be decomposed in the same two ways that the domain of integration of $Q_{a}$ is decomposed.

Since the $r$ integrals of (2.21) are analytical, then $Q_{b}$ can be written as

$$
Q_{b}=\int_{0}^{2 \sqrt{b^{2}-a^{2}}} d l \rho_{b}(l)
$$

where

$$
\rho_{b}(l)= \begin{cases}\frac{3\left(4 b^{2}-l^{2}\right)}{16\left(b^{3}-a^{3}\right)}, & 0 \leq l \leq(b-a) \\ \frac{3\left(b^{2}-a^{2}+2 l^{2}\right)\left(b^{2}-a^{2}\right)}{16\left(b^{3}-a^{3}\right) l^{2}}, & (b-a) \leq l \leq \sqrt{b^{2}-a^{2}} \\ \frac{12\left(b^{2}-a^{2}\right)-3 l^{2}}{16\left(b^{3}-a^{3}\right)}, & \sqrt{b^{2}-a^{2}} \leq l \leq 2 \sqrt{b^{2}-a^{2}} .\end{cases}
$$

Since $Q=Q_{a}+Q_{b}$, and $Q_{a}$ and $Q_{b}$ are given by (2.14) and (2.22) respectively, then $Q$ can be written as

$$
Q=\int_{0}^{2 \sqrt{b^{2}-a^{2}}} \rho_{l} d l
$$

where

$(2.25) \rho_{l}= \begin{cases}\frac{3\left(4 b^{2}-l^{2}\right)}{16\left(b^{3}-a^{3}\right)}+\frac{3 a^{2}}{4\left(b^{3}-a^{3}\right)}, & 0 \leq l \leq(b-a) \\ \frac{3\left(b^{2}-a^{2}+2 l^{2}\right)\left(b^{2}-a^{2}\right)}{16\left(b^{3}-a^{3}\right) l^{2}}+\frac{3\left(b^{2}-a^{2}-l^{2}\right)^{2}}{16\left(b^{3}-a^{3}\right) l^{2}}, & (b-a) \leq l \leq \sqrt{b^{2}-a^{2}} \\ \frac{12\left(b^{2}-a^{2}\right)-3 l^{2}}{16\left(b^{3}-a^{3}\right)}, & \sqrt{b^{2}-a^{2}} \leq l \leq 2 \sqrt{b^{2}-a^{2}},\end{cases}$

is the distribution of truncated chord lengths of the shell.

2.1. The Average Path Length of a Spherical Shell. The average path length $\langle l\rangle$ in a spherical shell is

$$
<l>\equiv \int_{0}^{\infty} \rho_{l} l d l .
$$

The substitution of (2.25) into (2.26) with a bit of algebra gives

$$
<l>=\frac{3(b-a)^{2}}{16\left(b^{3}-a^{3}\right)}\left(4 b^{2}+6 a b+4 a^{2}+(b+a)^{2} \log _{e} \frac{b+a}{b-a}\right) .
$$


3. The Distribution of Complete Chord Lengths of a Spherical Shell. We can derive the distribution of complete chord lengths in the same way that we derived the distribution of truncated chord lengths in the previous section. The only change that we need to make in the derivation is in the definition of the length of a chord. In order to be complete, we assume that the interior point of a spherical shell are equally probable and that the chords are isotropically distributed. So $\rho_{r}$ and $\rho_{\mu}$ are given by (2.1) and (2.2) respectively. We see that there two kinds of complete chords in the right figure of Fig. 1. The first type is entirely within the shell, while the second type crosses the hollow region of the shell. If $\mu$, the 'angle of inclination' of the chord with respect the vector $\mathbf{r}$, is between $-\sqrt{1-a^{2} / r^{2}}$ and $\sqrt{1-a^{2} / r^{2}}$, then the chord is the first type. If $|\mu|>\sqrt{1-a^{2} / r^{2}}$, then the chord is the second type. The chord length of the first type and the chord length of the second type are respectively

$$
\begin{array}{ll}
l_{a}=2 \sqrt{b^{2}-\left(1-\mu^{2}\right) r^{2}}, & 0 \leq|\mu| \leq \sqrt{1-a^{2} / r^{2}} \\
l_{b}=2 \sqrt{b^{2}-\left(1-\mu^{2}\right) r^{2}}-2 \sqrt{a^{2}-\left(1-\mu^{2}\right) r^{2}}, & \sqrt{1-a^{2} / r^{2}}<|\mu| \leq 1 .
\end{array}
$$

In the last section we broke the $\mu$ integral of $Q$ into 2 regions. In this section, we break the $\mu$ integral of $Q$ into 4 regions according to the breakpoints of (3.1), i.e.

$$
\begin{aligned}
Q & =\int_{a}^{b} d r \rho_{r} \int_{-1}^{1} d \mu \rho(\mu \mid r) \\
& =\int_{a}^{b} d r\left\{\int_{-1}^{-\sqrt{1-a^{2} / r^{2}}}+\int_{-\sqrt{1-a^{2} / r^{2}}}^{0}+\int_{0}^{\sqrt{1-a^{2} / r^{2}}}+\int_{\sqrt{1-a^{2} / r^{2}}}^{1}\right\} d \mu \rho(\mu \mid r) \\
& =2 \int_{a}^{b} d r \rho_{r} \int_{0}^{\sqrt{1-a^{2} / r^{2}}} d \mu \rho(\mu \mid r)+2 \int_{a}^{b} d r \rho_{r} \int_{\sqrt{1-a^{2} / r^{2}}}^{1} d \mu \rho(\mu \mid r),
\end{aligned}
$$

where we took into account that the $\mu$ integrals are symmetric with respect to $-\mu$. Since $Q$ is broken into two pieces, we can write it as $Q=Q_{a}+Q_{b}$, where

$$
\begin{aligned}
Q_{a} & \equiv 2 \int_{a}^{b} d r \rho_{r} \int_{0}^{\sqrt{1-a^{2} / r^{2}}} d \mu \rho(\mu \mid r), \\
Q_{b} & \equiv 2 \int_{a}^{b} d r \rho_{r} \int_{\sqrt{1-a^{2} / r^{2}}}^{1} d \mu \rho(\mu \mid r) .
\end{aligned}
$$

The chord lengths for $Q_{a}$ are given by the first equation of (3.1), and the chord lengths for $Q_{b}$ are given by the second equation of (3.1).

For $Q_{a}$, we change the variable of integration from $\mu$ to $l_{a}$. Solving the first equation of (3.1) for $\mu$,

$$
\mu=-\frac{\sqrt{\left(\frac{l_{a}}{2}\right)^{2}+r^{2}-b^{2}}}{r},
$$

and its derivative is

$$
\frac{d \mu}{d l_{a}}=\frac{\frac{l_{a}}{2}}{2 r \sqrt{\left(\frac{l_{a}}{2}\right)^{2}+r^{2}-b^{2}}}
$$




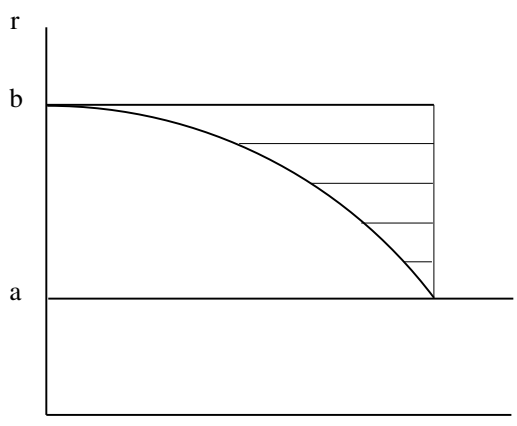

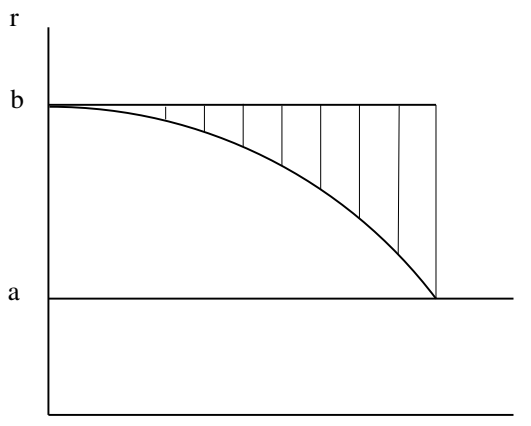

1

FIG. 3.1. The domain of integration of $Q_{a}$ for complete chords.

The substitution of (3.6) into (3.3) gives

$$
Q_{a}=2 \int_{a}^{b} d r \rho_{r} \int_{2 \sqrt{b^{2}-r^{2}}}^{2 \sqrt{b^{2}-a^{2}}} d l_{a} \rho(\mu \mid r) \frac{\frac{l_{a}}{2}}{2 r \sqrt{\left(\frac{l_{a}}{2}\right)^{2}+r^{2}-b^{2}}}
$$

Figure 4 shows the the domain of integration and the limits of the double integral of (3.7). When the order of integration is reversed, Fig. 4 shows that the integral of (3.7) can be written as

$$
\begin{aligned}
Q_{a} & =\int_{0}^{2 \sqrt{b^{2}-a^{2}}} d l_{a} \int_{\sqrt{b^{2}-\left(l_{a} / 2\right)^{2}}}^{b} d r \rho_{r} \rho(\mu \mid r) \frac{\frac{l_{a}}{2}}{r \sqrt{\left(\frac{l_{a}}{2}\right)^{2}+r^{2}-b^{2}}} \\
& =\int_{0}^{2 \sqrt{b^{2}-a^{2}}} d l \frac{3 l^{2}}{8\left(b^{3}-a^{3}\right)}
\end{aligned}
$$

For $Q_{b}$, we change the variable of integration from $\mu$ to $l_{b}$. Solving the second equation of (3.1) for $\mu$,

$$
\mu=\frac{\sqrt{\left(\left(l_{b} / 2\right)^{2}+2 r^{2}-b^{2}-a^{2}\right)^{2}+4\left(b^{2}-r^{2}\right)\left(r^{2}-a^{2}\right)}}{r l_{b}},
$$

we get

$$
\frac{d \mu}{d l_{b}}=\frac{\left(l_{b} / 2\right)^{4}-\left(b^{2}-a^{2}\right)^{2}}{r l_{b}^{2} \sqrt{\left(\left(l_{b} / 2\right)^{2}+2 r^{2}-b^{2}-a^{2}\right)^{2}+4\left(b^{2}-r^{2}\right)\left(r^{2}-a^{2}\right)}} .
$$

The substitution of (3.10) into (3.4) gives

$$
\text { (3.11) } Q_{b}=\int_{a}^{b} d r \int_{2(b-a)}^{2 \sqrt{b^{2}-a^{2}}} d l_{b} \frac{2 \rho_{r} \rho(\mu \mid r)\left(\left(b^{2}-a^{2}\right)^{2}-\left(l_{b} / 2\right)^{4}\right)}{r l_{b}^{2} \sqrt{\left(\left(l_{b} / 2\right)^{2}+2 r^{2}-b^{2}-a^{2}\right)^{2}+4\left(b^{2}-r^{2}\right)\left(r^{2}-a^{2}\right)}} .
$$

Since the integration limits of the inner integral in the above equation are independent of $r$, we can change the order of integration without changing the limits of integration. 
Furthermore the $r$ integral is analytical, so we get

$$
Q_{b}=\int_{2(b-a)}^{2 \sqrt{b^{2}-a^{2}}} d l \frac{3}{2\left(b^{3}-a^{3}\right)}\left(\frac{\left(b^{2}-a^{2}\right)^{2}}{l^{2}}-\frac{l^{2}}{16}\right) .
$$

Combining (3.8) and (3.12), we get

$$
Q=\int_{0}^{2 \sqrt{b^{2}-a^{2}}} d l \frac{3 l^{2}}{8\left(b^{3}-a^{3}\right)}+\int_{2(b-a)}^{2 \sqrt{b^{2}-a^{2}}} d l \frac{3}{2\left(b^{3}-a^{3}\right)}\left(\frac{\left(b^{2}-a^{2}\right)^{2}}{l^{2}}-\frac{l^{2}}{16}\right)
$$

Therefore the distribution of the complete chord lengths in a spherical shell is

$$
\rho(l)= \begin{cases}\frac{3 l^{2}}{8\left(b^{3}-a^{3}\right)}, & 0 \leq l \leq 2(b-a) \\ \frac{9 l^{2}}{32\left(b^{3}-a^{3}\right)}+\frac{12\left(b^{2}-a^{2}\right)^{2}}{8 l^{2}\left(b^{3}-a^{3}\right)}, & 2(b-a) \leq l \leq 2 \sqrt{b^{2}-a^{2}} .\end{cases}
$$

3.1. Average Complete Chord Length in a Spherical Shell. The average length $\langle l\rangle$ of a complete chord in a spherical shell can be determined easily from the density (3.14) and the formula

$$
<l>\equiv \int_{0}^{\infty} \rho_{l} l d l
$$

The substitution of (3.14) into (3.15) gives

$$
<l>=\frac{3(b-a)^{4}+9\left(b^{2}-a^{2}\right)^{2}}{8\left(b^{3}-a^{3}\right)}+\frac{3\left(b^{2}-a^{2}\right)^{2}}{4\left(b^{3}-a^{3}\right)} \log _{e} \frac{b+a}{b-a} .
$$

4. Acknowledgments. I would like to thank Bill Moran for introducing me to this subject, and I would like to thank David Miller for his insights into this problem.

\section{REFERENCES}

[1] P. A. M. Dirac, Approximate rate of neutron multiplication for a solid of arbitrary shape and uniform density, I: General Theory., MS.D.5, PartI, August 1943, 11, p. 1117-1128.

[2] P. A. M. Dirac, Approximate rate of neutron multiplication for a solid of arbitrary shape and uniform density, II: Application to the oblate spheroid, hemisphere and oblate hemispheroid., MS.D.5, PartI, August 1943, 11, p. 1131-1147. 